\title{
1. Remote Teaching of Programming in Mathematica: Lessons Learned
}

\author{
${ }_{2}$ Samuel T. Cahill, Patrick E. Bergstrom Mann, Andrew F. Worrall, and Malcolm I. Stewart*
}

Cite This: https://dx.doi.org/10.1021/acs.jchemed.0c00684

Read Online

3 ABSTRACT: The COVID-19 pandemic has resulted in a need for 4 social distancing measures in public spaces and will have an 5 important impact on university level teaching going forward. 6 Remote methods to complete laboratory related activities are a 7 potential means of students achieving practical credit while still 8 isolating or social distancing. We outline the implementation of 9 two novel remote exercises, based on coding in Mathematica, and 10 the methods used to provide ongoing help to students. These 11 exercises are used as a case study to identify potential problems 12 with remote exercises based on student feedback. Suggestions for how these problems might be overcome in further iterations of 13 these remote exercises are explored.

14 KEYWORDS: First-Year Undergraduate/General, Second-Year Undergraduate, Physical Chemistry, Computer-Based Learning, 15 Distance Learning/Self Instruction, Thermodynamics, Kinetics, Quantum Chemistry

\section{$16 \square$ INTRODUCTION}

17 The 2020 COVID-19 global pandemic has had a dramatic and 18 rapid effect on universities in the UK and across the globe. ${ }^{1}$ 19 The Universities UK issued framework for a safe and successful 20 emergence from lockdown includes the need for "changes to 21 university $\cdots$ infrastructure" and "flexibility in place to $\cdots$ support 22 students to achieve their learning outcomes in a safe manner". ${ }^{2}$ 23 While some universities have already come forward with 24 decisions to hold remote lectures to alleviate the spread of 25 infection, ${ }^{3}$ there is a substantial responsibility for teaching 26 laboratories to follow suit in reducing contact between 27 students while still providing elements of practical teaching. ${ }^{4}$

28 Because of its term structure, our department has been 29 running a remote laboratory course from 26th April to present 30 (15th June). While the teaching laboratory has been out of 31 bounds, remote, computer-based, teaching has provided the 32 opportunity to focus on other important and transferable skills 33 such as data analysis and the introduction of coding 34 languages. ${ }^{5,6}$ The introduction of exercises based around 35 these topics has allowed students to continue to work toward 36 their practical credit requirement (in the UK, a four year 37 Masters' course must contain a minimum of $400 \mathrm{~h}$ of practical 38 work for accreditation by the Royal Society of Chemistry) ${ }^{7}$ but 39 also broadened the scope of what practical exercises the 40 laboratories may offer at the start of next academic year-with 41 more advanced and applied coding activities being possible 42 now that the students have a strong foundation.

43 It should be noted that the likely need for social distancing 44 in laboratories next academic year will reduce the number of 45 students that can be working in the lab at any time. The 46 continued use of computer-based exercises, alongside the 47 reintroduction of practical laboratories, will let more students undertake practical course work at the same time by moving 48 some of this work outside of the lab. In this way the student 49 throughput required for every student to meet their practical 50 quota of lab hours can still be achieved, despite the change in 51 circumstances.

In this communication we introduce two exercises, based in 53 Mathematica, that were made available to our first and second 54 year students. Our institution owns a site license for this 55 software so that all students are able to download and install 56 the application on their home computer and carry out these 57 exercises while in isolation. The first exercise consists of a 58 series of activities aimed at introducing syntax and useful 59 applications of Mathematica, while the second seeks to build 60 on this introduction through addressing some simple quantum 61 mechanical problems. (All material relating to these two novel 62 exercises are included as Supporting Information and are ready 63 to be implemented by others. Further details of the topics 64 covered can be found in Table 1.) To date, both of these $65 \mathrm{tl}$ exercises have been completed by 200 of our students, with a 66 further 68 just completing the introductory exercise. We have 67 used these exercises as a case study for lessons learned in 68 rolling out remote exercises and to suggest ways in which we 69 have, or other institutions might, learn from our initial trial. 70

Special Issue: Insights Gained While Teaching Chem-

istry in the Time of COVID-19

Received: June 16, 2020

Revised: July 14, 2020 
Table 1. Topics Covered in the Two Mathematica Exercises

\begin{tabular}{ll}
\multicolumn{1}{c}{ Practical Title } & \multicolumn{1}{c}{ Topics } \\
S217: & - Defining variables and expressions. \\
Introduction to & - Implementing text strings. \\
Mathematica & - Manipulating lists. \\
& - Employing in-built functions and defining novel \\
& functions. \\
& - Plotting data and functions. \\
& - Solving algebraic and differential equations. \\
& - Integrating and differentiating functions. \\
& - Linear regression and nonlinear curve fitting. \\
& - Plotting wave functions and probability distributions \\
& for particle-in-a-box, harmonic and Morse oscillator, \\
C214: Quantum & and hydrogen orbital problems. \\
Mechanics in & Exploring different visualizations of $2 \mathrm{D}$ probability \\
Mathematica & distributions. \\
& - Evaluating expectation values and uncertainties.
\end{tabular}

\section{INTRODUCING EXERCISES AND IMPLEMENTING 72 HELP FOR STUDENTS}

73 The two exercises, along with introductions detailing 74 instructions, credit hours, and methods for downloading, 75 installing, and running Mathematica (see Supporting Informa76 tion) were made available to students via our university's 77 virtual learning environment.

78 Given the potential for increased cognitive load when 79 working from home, the introductory practical (known as 80 S217) was constructed in a scaffolded manner with worked 81 examples throughout to help guide students comfortably 82 through the activity. ${ }^{8-10}$ The introduction of each new aspect 83 of code was accompanied by one or more exercises and 84 revisited in later activities through progressively more complex 85 tasks. The follow up quantum mechanics exercise (known as 86 C214) was written with reduced scaffolding and was designed 87 to allow students to put aspects of what they had learned from 88 the introduction into practice-there were potentially multiple 89 methods of achieving the correct answer and all methods 90 would be treated equally during assessment.

91 As was made clear in the introduction to both exercises, a 92 help system was implemented so that students could make 93 enquiries in the event that they had difficulties while working 94 through the exercises. Students were invited to send enquiries 95 via e-mail to a dedicated address to which all teaching staff had 96 access. Students were requested to title their e-mails with the practical code, for ease of administration, and to briefly 97 describe the nature of the problem, if possible. Students were 98 also instructed that short conversations via video conferencing 99 would be available if they felt that e-mail contact would not 100 prove sufficient to solve their problem. With our university 101 having a substantial number of international students in its 102 chemistry cohort, we took the decision not to timetable 103 exercises and to do our best to provide round-the-clock help to 104 facilitate those students working in other time zones or difficult 105 work environments.

\section{FEEDBACK RESULTS}

107

An anonymous survey was issued to students via our virtual 108 learning environment (see the Supporting Information for a 109 full list of questions). The main goal of our survey was to 110 ascertain how long students were taking to complete each 111 exercise and how they were finding the help available. Out of 112 268 students, 71 provided information about introductory 113 exercise, S217, while 53 of 200 provided feedback for C214 114 (due to anonymity of each survey, it is not possible to 115 determine the extent of overlap between the two sets of 116 respondents).

\section{Time Taken to Complete Exercises}

118

Our initial credit allocations for the two exercises were $12 \mathrm{~h}$ for 119 the introduction and $6 \mathrm{~h}$ for the follow up quantum mechanics 120 exercise. These allowances were based on feedback from 121 teaching assistants who had little, or no, experience in coding. 122 A distribution of hours taken for each exercise is provided in 123 Figure 1.

The feedback showed that $50 \%$ of the total student 125 respondents completed S217 within our estimated time (12 126 $\pm 3 \mathrm{~h}$ ), with $6 \%$ taking below $9 \mathrm{~h}$ and the remaining $44 \%$ of 127 students taking greater than $15 \mathrm{~h}$ to complete the exercise. The 128 results were more dramatic for the follow up exercise C214, 129 where only $32 \%$ of the students completed the exercise within 130 the estimated time $(6 \pm 3 \mathrm{~h})$ and the other $68 \%$ took greater 131 than $9 \mathrm{~h}$.

These results show that a sizable number of students were 133 taking longer than expected to complete the two exercises, too 134 many in the case of $\mathrm{C} 214$ (we would anticipate $50-60 \%$ of the 135 cohort to fall within a $\pm 3 \mathrm{~h}$ range). These results may be due 136 to difficulties completing the exercise and a lack of immediate 137 help from teaching assistants due to the remote learning 138
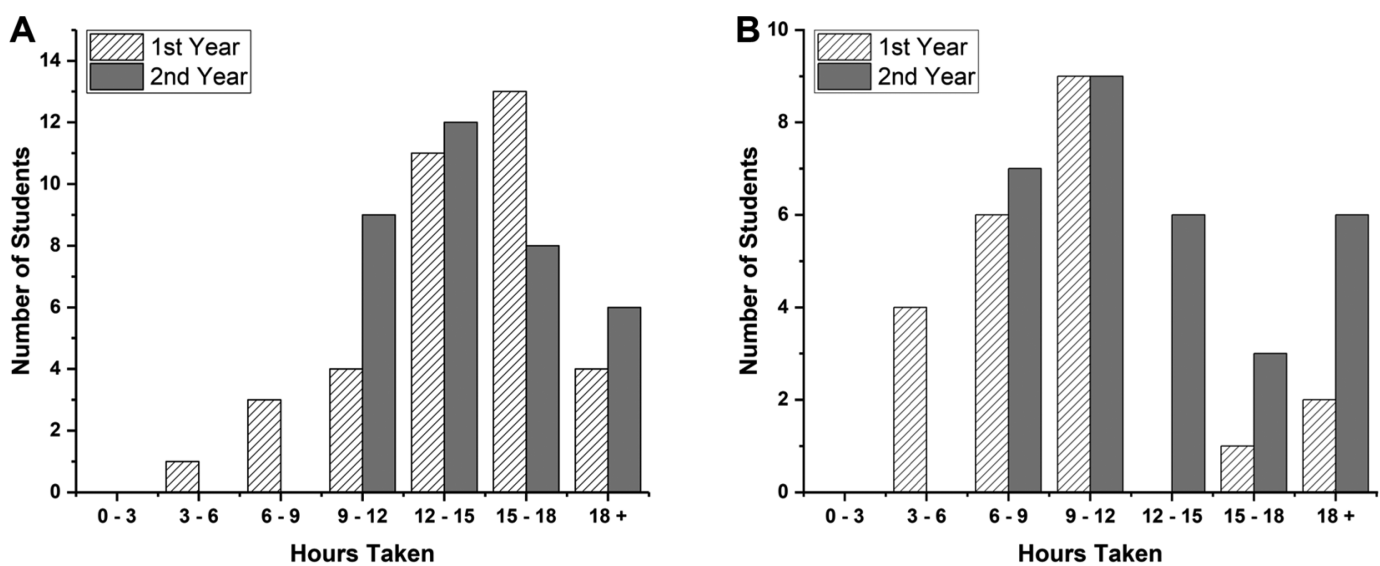

Figure 1. Distribution of hours taken to complete the Mathematica exercises. (A) Reported hours taken to complete the introductory exercise S217. (B) Reported hours taken to complete quantum mechanics exercise C214. 
139 environment (see below), or an increased cognitive load due to 140 the nonideal working environment. ${ }^{11,12}$

\section{Student Enquiries}

142 As stated above, remote help was provided to students 143 completing this exercise via email and video call (the latter on 144 request). Our team worked hard to ensure that waiting times 145 for responses to e-mail queries were kept to a minimum so as 146 to minimize any disruption to flow that the students might 147 experience. Of the students that responded to our survey, 38\% 148 contacted staff with help enquiries about S217 and 63\% 149 contacted with enquiries about C214. An outline of the topics

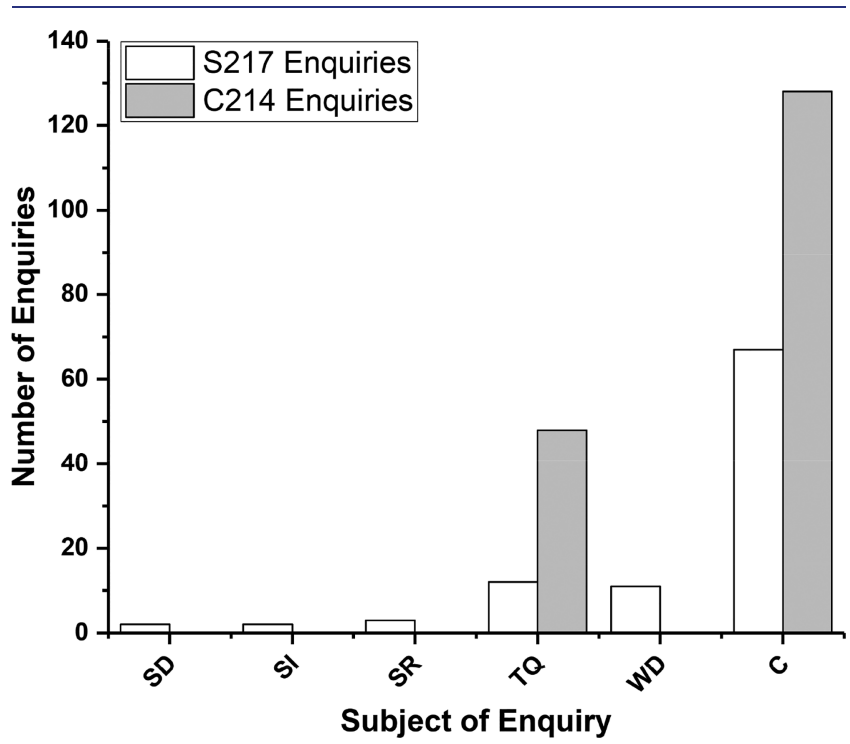

Figure 2. A summary of enquiries received via e-mail during the course of the term. Enquiries are categorized according to the particular problems encountered by the students: SD, software download; SI, software installation; SR, software registration; TQ theory or understanding of question; $\mathrm{WD}$, setting a working directory; $\mathrm{C}$, code syntax or implementation.

151 from technical difficulties such as software issues to theory 152 associated with chemical questions ( $3 \%$ and $22 \%$, respectively), 153 but most enquiries were specifically about aspects of coding or 154 use of the Mathematica software package (note that, due to its nature as a followup exercise, there were no download and 155 installation enquires relating to C214).

Other than general errors in syntax, the most common 157 coding problem in S217 was students not being able to set a 158 working directory (11\% of all enquiries relating to S217) - a 159 number of students seemed to have difficulty navigating 160 through general file structure or typing out folder addresses. 161 Errors in coding were spread across the five notebooks, with no 162 particular clustering of problems. For C214 there was a greater 163 portion of enquiries relating to theory (27\% of total C214 164 enquiries), with the most common being students forgetting 165 how to normalize wave functions or calculate probabilities 166 through integration. The greatest number of coding issues 167 related to typing out the full hydrogenic wave functions in the 168 third notebook; students were much more error prone in this 169 exercise and often struggled to debug what they had written- 170 perhaps a little surprising since students are simply required to 171 convert a mathematical formula to code (albeit a long one) 172 rather than implement any novel features.

We initially suspected that those students who sent e-mail 174 enquiries would complete the two exercises at a faster pace 175 since they would not get stuck troubleshooting their code 176 when it was passed to a teaching staff member. Figure 3 shows $177 \mathrm{f} 3$ that this is not really the case-the percentage of students 178 taking greater than $15 \mathrm{~h}$ to complete S217 was $44 \%$ and $43 \% 179$ for those sending enquiries and those not sending enquiries, 180 respectively. For $\mathrm{C} 214$ the numbers were the opposite of our 181 initial guess with $42 \%$ of students taking greater than $12 \mathrm{~h}$ to 182 complete the task when sending enquiries versus $16 \%$ of 183 nonenquiring students.

184

These results have led us to believe that students having 185 difficulties have only been contacting us for help as a last 186 resort, rather than at any time that they encounter an issue- 187 this result is backed up by students' written feedback (see 188 below), but stands in contrast to the information given 189 alongside these exercises as well as further e-mails sent from 190 the department emphasizing that students should get in touch 191 whenever they have any problems with computational 192 exercises. Another possibility is that students have a different 193 perception of the requirement for completion of the exercise: 194 in the lab, a student must leave at closing time regardless of 195 whether they have completed their practical or not, but there is 196 no cutoff time in these remote exercise unless the student 197 imposes it themselves.
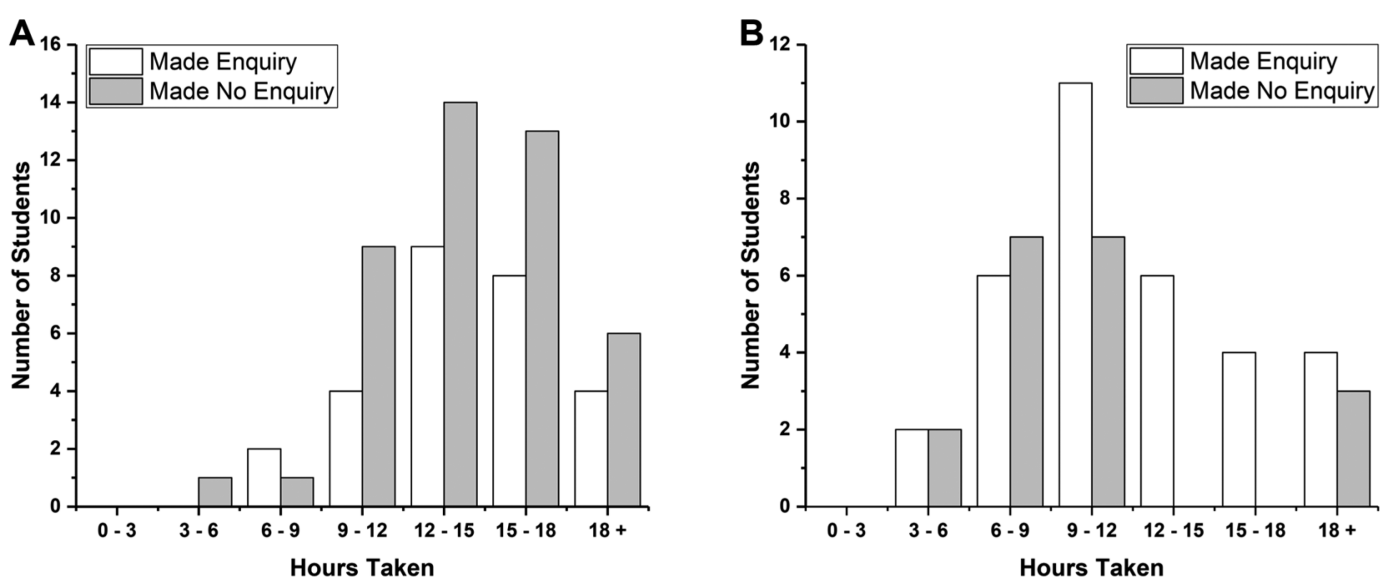

Figure 3. Distribution of hours taken to complete the Mathematica exercises separated by students who did or did not send e-mail enquiries. (A) Reported hours taken to complete S217. (B) Reported hours taken to complete C214. 


\section{Other Sources of Information}

200 The students who had not contacted us for assistance were 201 polled on other sources of information they had employed 202 when they had encountered problems. This information is 203 summarized in Figure 4. As seen, 29\% of responses indicated
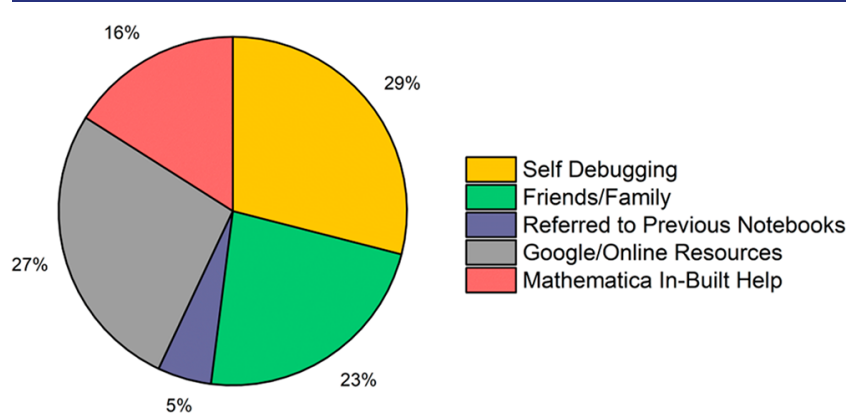

Figure 4. Other types of help students reported as methods to solve problems during the two exercises. Percentage of total responses that mentioned each of five major methods of troubleshooting.

204 that students were simply able to fix their problems through 205 taking the time to debug the code themselves with a further 5\% 206 of responses citing the previous introductory notebooks as a 207 useful reference. Further, 16\% of responses indicated that 208 Mathematica's in-built documentation was a useful guide, 209 while online searches and help from friends or family were the 210 most popular sources of information at $27 \%$ and $23 \%$ of 211 responses, respectively.

212 We believe that all of these sources of information are valid: 213 students are actively encouraged to browse the Mathematica 214 documentation in a couple of the exercises, and the ability to 215 search online for a solution to a coding problem is a regular 216 source of information for active programmers. One potential 217 concern is those students citing friends and family as a source 218 of help, while peer-based learning and collaboration are 219 important and useful methods in scientific teaching, ${ }^{13}$ there 220 is a fine line between collaboration and copying-something 221 that is particularly difficult to root out when scanning through 222 programming code. ${ }^{14}$

\section{Reasons for Not Making Contact When Needing 224 Assistance}

225 Some reasons for students not making enquiries are grouped 226 thematically in Table 2. Three common themes were that 227 students found e-mail communication difficult or were unable 228 to explain their problem in text, students thought that e-mail 229 responses would be too slow, and students felt unable to 230 contact with what they viewed as problems that were too small 231 about which to bother staff.

232 These responses are interesting given the information that 233 was provided to students about accessing help. Multiple 234 students said that e-mail was not a preferred method of 235 communication, but we thought we had made clear that video 236 calls were another option for seeking help. We have also 237 worked very hard to provide rapid responses to all e-mail 238 enquiries throughout the term (subject to time zone 239 differences), with response times of 5-10 min during working 240 hours and 30-60 min during evenings and weekends. 241 Evidently these times are not rapid enough for students, or 242 (if they are suitably fast) students are assuming that responses 243 will be slow and so have decided not to make an enquiry.
Table 2. Reasons for Students Not Contacting for Help via Email

Theme
$\begin{aligned} & \text { Difficulty with contact } \\ & \text { via e-mail }\end{aligned}$
"talking to someone one on one about a problem is
obviously much more useful than an email"
difficult to ask for help when you don't know what is
wrong"
- "I didn't think I could explain my issues in an email"
- "it feels difficult to explain the problem I am facing
via e-mail"
- "by the time I had emailed and received a reply in
that time I could probably do a google and find out"
- "emailing about every small question I had $\cdots$ would
have made the lab take a lot longer"
- "Tried to sort problems out myself and thought this
might be quicker than emailing"
- "wanted to get the practical done in a reasonable
time, rather than start-stopping and waiting for a
response"
- "I found myself stuck most because of errors that
seemed too uncomplicated to ask someone about
them"
- "I did not want to email the CTL until I'd exhausted
all other options"

We find the last category of reasons more troubling. In a 244 laboratory setting, teaching assistants are able to look over the 245 shoulder of students, actively providing help when they are 246 having difficulties, and keeping the weaker students in step 247 with the cohort so that they complete their exercise in a 248 sensible time. ${ }^{15}$ With remote exercises, this is not possible and 249 the onus is solely on students to ask for help. Should students 250 decide that their problems are not sufficient to bother teaching 251 staff, it is near impossible to provide help in a remote teaching 252 context and students will likely take a lot longer to complete 253 exercises.

\section{DISCUSSION}

We have successfully implemented two remote exercises using 256 Mathematica which, at the time of writing, have been 257 completed by more than 200 students across the first and 258 second year cohort. While the majority of students have 259 successfully completed all of the outlined tasks with a good 260 quality of work, we have found that the time taken for students 261 to complete these exercises has substantially exceeded 262 expectations. In the case of our second exercise, C214, we 263 have re-evaluated the credit allowance for this exercise from 6264 to $12 \mathrm{~h}$, based on student feedback.

265

We believe that the time taken by students to complete 266 these exercises has been increased due to a greater cognitive 267 load generated by the need to work from home, but is also the 268 product of a lack of active feedback from teaching staff, which 269 comes as a consequence of remote working. While we have 270 manned an enquiry email address and fielded video calls to 271 help many students when they have had difficulty, this is reliant 272 on students actively seeking help. Our concern is that those 273 students who need the most help to complete exercises in a 274 timely manner may not be the ones to seek help themselves. In 275 a teaching lab environment these students would typically be 276 identified and assisted throughout a session to make sure that 277 they complete an exercise on time. Reasons for students not 278 making contact include difficulties with communication by e- 279 mail, an assumption that responses to e-mail enquiries are, or 280 will be, too slow, or that their problems are too small to 281 warrant bothering teaching staff. 
283 To try to increase our help for struggling students, and 284 students in general, if further remote laboratory teaching needs 285 to be implemented in the future we propose the following 286 modifications to the help provided this term: 304 writing emails formal and impersonal will be more likely to 305 contact us for help and with any problem they might have. In 306 this way we would aim to increase the help available to all 307 students, increase their productivity, and bring the time taken 308 for exercise completion more in line with our expectation. By 309 aligning our proposed credit hours more with the time that 310 students take to complete each exercise we should maintain a 311 greater student focus and, hence, make these activities greater 312 teaching aids through improved student engagement. ${ }^{16,17}$

\section{ASSOCIATED CONTENT}

\section{SI Supporting Information}

315 The Supporting Information is available at https://pubs.ac316 s.org/doi/10.1021/acs.jchemed.0c00684.

317 Introduction to Mathematica, complete notes (PDF)

318 Introduction to Mathematica, student notebooks (ZIP)

319 Quantum Mechanics in Mathematica, complete notes $320 \quad$ (PDF)

321 Quantum Mechanics in Mathematica, student note322 books (ZIP)

323 Survey questions (PDF)

\section{AUTHOR INFORMATION}

325 Corresponding Author

326 Malcolm I. Stewart - Chemistry Teaching Laboratory, 327 Department of Chemistry, University of Oxford, Oxford OX1 328 3PS, United Kingdom; @ orcid.org/0000-0002-5724-9160; 329 Email: malcolm.stewart@chem.ox.ac.uk

\section{Authors}

331 Samuel T. Cahill - Chemistry Teaching Laboratory, 332 Department of Chemistry, University of Oxford, Oxford OX1 333 3PS, United Kingdom; (1) orcid.org/0000-0002-0929-4186

334 Patrick E. Bergstrom Mann - Chemistry Teaching Laboratory, 335 Department of Chemistry, University of Oxford, Oxford OX1 336 3PS, United Kingdom; (ㄷ) orcid.org/0000-0003-1428-1440

337 Andrew F. Worrall - Chemistry Teaching Laboratory, 338 Department of Chemistry, University of Oxford, Oxford OX1 339 3PS, United Kingdom; (1) orcid.org/0000-0002-2875-6905

340 Complete contact information is available at: https://pubs.acs.org/10.1021/acs.jchemed.0c00684

Notes

The authors declare no competing financial interest.

REFERENCES

(1) Montacute, R.; Holt-White, E. COVID-19 and Social Mobility 345 Impact Brief \#2: University Access \& Student Finance; The Sutton 346 Trust, 2020.

(2) Universities UK. Principles and Considerations: Emerging from 348 Lockdown; Universities UK, 2020.

(3) Fazackerley, A. Forget Freshers' Week: Universities Prepare to 350 Teach New First Years Online. The Guardian, April 3, 2020.

(4) HM Government UK. Working Safely during COVID-19 in Labs 352 and Research Facilities; Gov.UK, 2020.

(5) Overton, T.; McGarvey, D. J. Development of Key Skills and 354 Attributes in Chemistry. Chem. Educ. Res. Pract. 2017, 18 (3), 401- 355 402.

(6) Weiss, C. J. Scientific Computing for Chemists: An Under- 357 graduate Course in Simulations, Data Processing, and Visualization. J. 358 Chem. Educ. 2017, 94 (5), 592-597.

(7) Accreditation of Degree Programmes; Royal Society of Chemistry, 360 2019.

(8) Paas, F.; Renkl, A.; Sweller, J. Cognitive Load Theory and 362 Instructional Design: Recent Developments. Educ. Psychol. 2010, 38, 363 $1-4$.

(9) Chen, O.; Woolcott, G.; Sweller, J. Using Cognitive Load 365 Theory to Structure Computer-Based Learning Including MOOCs. J. 366 Comput. Assist. Learn. 2017, 33 (4), 293-305.

(10) Impelluso, T. J. Assessing Cognitive Load Theory to Improve 368 Student Learning for Mechanical Engineers. Am. J. Distance Educ. 369 2009, 23 (4), 179-193.

(11) Chandler, P.; Sweller, J. Cognitive Load Theory and the 371 Format of Instruction. Cogn. Instr. 1991, 8 (4), 293-332.

(12) Stiller, K. D.; Bachmaier, R. Cognitive Loads in a Distance 373 Training for Trainee Teachers. Front. Educ. 2018, 3, 44.

(13) Chan, C.; Van Aalst, J., Learning, Assessment and 375 Collaboration in Computer-Supported Environments. In What We 376 Know About CSCL. Computer-Supported Collaborative Learning Series; 377 Strijbos, J. W., Kirschner, P. A., Martens, R. L., Eds.; Springer, 2004; 378 pp 87-112.

(14) Albluwi, I. Plagiarism in Programming Assessments: A 380 Systematic Review. ACM Trans. Comput. Educ. 2019, 20 (1), 6.

(15) Chang, J. L.; Piket-May, M.; Avery, J. P. Using Active Student 382 Feedback in the Learning Environment. FIE '98. 28th Annual Frontiers 383 in Education Conference. Moving from 'Teacher-Centered' to 'Learner- 384 Centered' Education; IEEE, 1998; Vol. 2, pp 643-646. 385

(16) Smith, K. C.; Alonso, V. Measuring Student Engagement in the 386 Undergraduate General Chemistry Laboratory. Chem. Educ. Res. Pract. 387 2020, 21 (1), 399-411.

(17) Galloway, K. R.; Bretz, S. L. Development of an Assessment 389 Tool To Measure Students' Meaningful Learning in the Under- 390 graduate Chemistry Laboratory. J. Chem. Educ. 2015, 92 (7), 1149- 391 1158. 